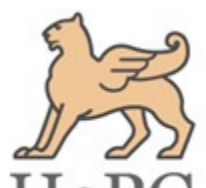

\title{
Acute consumption of Peppermint and Chamomile teas produce contrasting effects on cognition and mood in healthy young adults
}

\author{
Mark Moss $^{1}$, Robert Jones ${ }^{1}$, Lucy Moss ${ }^{1}$, Richard Cutter ${ }^{1}$ and Keith Wesnes ${ }^{1,2}$ \\ ${ }^{1}$ Department of Psychology, Northumbria University, Newcastle upon Tyne, NE1 8ST, UK \\ ${ }^{2}$ Wesnes Cognition, http://www.wesnes.com
}

Article history

Received: 25 June 2016

Accepted: 10 August 2016

Published: 30 September 2016

(C) Moss et al. (2016)

Special Section

Target constituents in medicinal, aromatic and food plants

Section Editor

Alessandra Bertoli

\section{Publisher}

Horizon e-Publishing Group

Corresponding Author

Mark Moss

国绎.moss@northumbria.ac.uk

\begin{abstract}
This study aimed to assess the acute effects of Peppermint and Chamomile herbal teas on cognitive performance and mood in healthy young adults. A single factor independent groups design was employed. One hundred and eighty undergraduate students volunteered to take part in the study for which they received course credit. Participants were randomly allocated to one of three treatments: Peppermint tea, Chamomile tea or hot water (Control). Mood scales were completed and participants then consumed their drink over a ten minute period and rested for twenty minutes. Cognitive performance was assessed using a tailored version of The Cognitive Drug Research (CDR) computerised assessment system. Post testing mood scales were then completed. Data were analysed using independent groups ANOVAs followed by Tukey post hoc comparisons. The analysis revealed that Peppermint tea significantly improved long term memory and speed of memory compared to both Chamomile and control treatments. Chamomile tea significantly slowed speed of attention and impaired working memory compared to the Peppermint treatment. Peppermint tea significantly increased subjective alertness compared to the Chamomile and control conditions. Chamomile significantly increased subjective calmness compared to the Peppermint treatment. The data show that acute consumption of Peppermint and Chamomile teas can impact on cognition and mood in healthy adults in contrasting directions. The enhancing and arousing effects of Peppermint and calming/sedative effects of Chamomile observed are in keeping with the purported properties of these herbs and suggest beneficial effects can be drawn from their use.
\end{abstract}

Keywords

Chamomile; Peppermint; Cognition; Memory; Mood

Moss, M., R. Jones, L. Moss, R. Cutter and K. Wesnes. 2016. Acute consumption of Peppermint and Chamomile teas produce contrasting effects on cognition and mood in healthy young adults. Plant Science Today 3(3): 327-336. http://dx.doi.org/10.14719/pst.2016.3.3.246

\section{Introduction}

Herbal based preparations have been used in promotion of health and the treatment of disease for as long as written records exist (Vickers and Zollman, 1999) and their popularity continues unabated by the development of modern pharmaceutical science and medicine (Bent and Ko,
2004). Worldwide, it is estimated that as much as $65 \%$ of the population rely on such traditional preparations as the first point of treatment (Ernst, 2000). Two herbs that represent a significant share of the market for herbal infusions in Europe are Peppermint (Mentha piperita) and German chamomile (Matricaria recutita). Peppermint has a 
long standing reputation as a therapeutic intervention for irritable bowel syndrome, nausea and tuberculosis and recent research offers some support to the received wisdom of ages. A systematic review concluded that peppermint treatment offered significant benefit compared to placebo for the treatment of irritable bowel syndrome (Ford et al., 2008) with effect sizes comparable to conventional treatments. Postoperative nausea has been demonstrated to be reduced and associated analgesia enhanced in a placebo controlled study (Tate, 1997), and tuberculosis inflammation has been shown to be reduced by the inhalation of peppermint aroma in a manner consistent with reduced recurrence and seriousness of the decay cavities (Shkurupil et al., 2006). The essential oil of Peppermint includes a number of potentially active volatile compounds including menthol which has been identified as exerting its antiemetic effect via the serotonin receptor ion-channel complex (Heimes et al., 2011). Peppermint also increases ambulatory activity in animal models, indicating the ability to be arousing; an effect potentially mediated by dopamine pathways (Umezu and Morita, 2003). Furthermore, Jasira et al. (2013) reported enhanced memory in rats assessed using T-maze and radial arm maze tasks following oral administration of peppermint oil, effects they put down to increased brain perfusion. In the arena of human behaviour, oral administered peppermint has been demonstrated to improve exercise performance (Meamarbashi and Rajabi, 2013b). The authors suggest that increased neural oxygenation as a consequence of enhanced ventilation through the relaxation of bronchial smooth muscles, and a reduction in plasma lactate levels are the most likely explanations of this enhancement. The impact of Peppermint on psychological variables has largely been approached from an aroma perspective where it has been shown to alleviate pain and reduce depression in arthritis patients (Kim et al., 2005), whilst studies involving healthy participants have demonstrated the potential for enhancement effects. In a study of prolonged simulated driving peppermint aroma inhalation produced increased ratings of alertness, decreased ratings of task demands, anxiety and fatigue, and lessened frustration over the driving simulation procedure (Raudenbush et al., 2009). An investigation into the possible effects of aromas on cognition in healthy young adults revealed that Peppermint improved subjective alertness and enhanced memory (Moss et al., 2008), an effect that may be a consequence of the inhibition of acetylcholinesterase (Kulišić-Bilušić et al., 2008).

The Chamomile plant is to be found across much of Europe and Western Asia, and its flowers have been used to prepare teas, produce tablets, or applied as oils to promote relaxation and induce sleep for hundreds of years (Mills and Bone, 2000; Sánchez-Ortuño et al., 2009). In a study of healthy adults reporting no sleep problems those receiving chamomile jelly reported higher ratings of relaxation, lower sleep onset latency, night-time wakefulness, and increased morning sleepiness compared to placebo (Kakuta et al., 2007). Although the neural mechanism is at this time unclear, studies suggest that the sedative effects result from modulation of y-aminobutryic acid (GABA) receptors (Viola et al., 1995). Chamomile tea has also been commonly employed in the treatment of digestive problems, diabetes and osteoporosis (Srivastava et al., 2010), and for the promotion of health by boosting the immune system (Wang et al., 2005) and reducing blood pressure (Zeggwagh et al., 2009), although further studies are required to firmly establish the validity of these latter effects. Psychologically, Chamomile has been used for the treatment of generalised anxiety disorder (Amsterdam et al., 2009), and via aromatherapy massage for the treatment of agitation and behavioural disturbances in dementia, with somewhat mixed effects (Burleigh and Armstrong, 1997). Exposure to the ambient aroma of chamomile essential oil has been shown to impair memory and increase calmness in young adults (Moss et al., 2006), in a study that interestingly also indicated that the impact of the aroma could be changed by manipulating participants' expectancies.

The current study aimed to extend our knowledge regarding herbal infusions by assessing the potential for acute ingestion of Peppermint and Chamomile teas to affect cognition and mood in healthy young adults. Reputation and previous literature cited above suggest that these two herbs will have opposite effects on both areas. Peppermint might be expected to increase alertness and positively influence memory, whereas Chamomile might increase calmness and impair memory. The extent to which any effects observed for mood and cognition correlate will help inform the debate around the mechanisms underpinning the cognitive effects of herbs and aromas previously reported.

\section{Methods \\ Design}

A one factor independent groups design was employed. The independent variable was condition and had three levels, Chamomile tea, Peppermint tea or hot water (control). The dependent variables were the scores from the CDR computerised cognitive assessment system and self-reported mood.

\section{Participants}

One hundred and eighty undergraduate students participated in the study for course credit. Participants were randomly assigned to experimental condition using a list of random numbers. The control condition was made up of 
38 females (mean age $=22.1$ years, $\mathrm{SD}=3.8$ ) and 22 males (mean age $=22.2$ years, SD 4.0). The Peppermint tea condition was made up of 36 females (mean age $=22.1$ years, $\mathrm{SD}=4.3$ ) and 24 males (mean age $=21.9$ years, $\mathrm{SD}=3.4$ ). The Chamomile tea condition was made up of 41 females (mean age $=22.6$ years, $\mathrm{SD}=4.8$ ) and 19 males (mean age $=24.32, \mathrm{SD}=5.3$ ).

\section{Materials}

\section{Teas}

'Clipper Teas' (Kallo Foods Ltd, River View, 2 The Meadows Business Park, Station Approach, Blackwater, Camberley, Surrey, GU17 9AB) organic Peppermint and Chamomile infusions were used to produce the teas. Each participant received a $200 \mathrm{ml}$ cup of tea served in a white ceramic mug. The tea was brewed in the mug with boiling water for 5 minutes and the bag removed prior to consumption. Cold water was available for the participants to cool the tea to their taste. Participants were instructed to consume the tea slowly over a ten minute period. Participants in the control condition were provided with $200 \mathrm{ml}$ of water that had been boiled and left to stand in a mug for 5 minutes and subsequently cooled with cold water to the participants' taste. This was then consumed slowly over a ten minute period.

\section{Cognitive Measures}

A tailored version of the Cognitive Drug Research (CDR) computerised assessment system was employed to evaluate cognitive performance. The CDR system includes a number of measures that are specific to particular aspects of attention, working memory and long term memory and has been shown to be sensitive to the effects of herbal extracts (Kennedy et al., 2000, 2001; Scholey et al., 2008) and the aromas of essential oils (Moss et al., 2003, 2008). Stimuli are presented on a colour monitor, and (with the exception of word recall) responses are made using a simple response module containing two buttons labelled 'Yes' and 'No' respectively. A suite of programmes controls all aspects of testing, including selection of appropriate sets of stimuli for presentation and recording all responses.

The tests employed in this study were presented in the following order:

Word presentation. A series of 15 words is presented sequentially for one second each with an inter-stimulus interval of one second. The words are a mix of one two and three syllables.

Immediate Word Recall. The computer display counts down sixty seconds during which time participants write down as many of the words from the list as possible. Recall is scored for number of correct words, and errors (words not presented in the list).
Picture Presentation. Twenty photographs are presented, with a stimulus duration of 2 seconds each, and inter stimuli interval of 1 second.

Simple Reaction Time. The word Yes is presented in the centre of the screen. The participant has to press the Yes button as quickly as possible. There are 50 trials and the intertrial interval varies randomly between 1 and 2.5 seconds. The reaction time is recorded in msec.

Digit Vigilance. A number is displayed constantly to the right of the screen. A series of 240 digits is presented one at a time in the centre at a rate of 80 per minute; 45 match the constantly displayed digit. The participant has to press the Yes button as quickly as possible every time the digit in the centre matches the one constantly displayed. Accuracy of response (\%), reaction time (msec), and number of false alarms are recorded.

Choice Reaction Time. Either the word Yes or the word No is presented in the centre of the screen. The participant has to press the Yes or No button as appropriate and as quickly as possible. There are 30 trials (25 'Yes' and 25 'No') and the intertrial interval varies randomly between 1 and 2.5 seconds. Accuracy (\%) and reaction time (msec) are recorded.

Spatial Working Memory. A schematic picture of a house is presented for 5 seconds. The house has nine windows in a $3 \times 3$ pattern, four of which are illuminated. A series of 36 presentations of the same house in which just one window is illuminated follow, and the participant has to respond Yes if the window was one of the four lit in the original presentation, or No if it was not. Sixteen of the stimuli require a Yes response and 20 a No response. Reaction time and accuracy are recorded and a sensitivity index calculated.

Memory Scanning. Five digits are presented singly at the rate of one every second for the participant to remember. A series of thirty digits is then presented. For each, the participant must press Yes or No according to whether the digit is thought to be one of the five presented initially. Fifteen stimuli require a Yes response and 15 a No response. This is repeated three times using a different 5 digits on each occasion. Reaction time is recorded and a sensitivity index calculated.

Delayed Word Recall. The computer counts down sixty seconds during which time participants free recall as many of the words from the list as possible. Recall is scored for number of correct words; and errors (words not presented in the list).

Word Recognition. The 15 words initially presented for the word recall are presented again in random order interspersed with 15 new words. The participant presses Yes or No each time to signal whether or not the word was from the original list. Reaction time and accuracy are recorded and a sensitivity index calculated.

Picture Recognition. The 20 pictures presented earlier are shown again in random order 
interspersed with 20 similar new ones. The participant signals recognition by pressing the Yes or No button as appropriate. Reaction time and accuracy are recorded and a sensitivity index calculated.

'Pencil and Paper' Visual Analogue Scales, assessing subjective levels of alertness, calmness and contentedness, were presented prior to and following the computerised tests. Participants are required to indicate their current state by marking a line drawn between two bipolar adjectives. Subjective ratings of pleasantness and strength of ambient aroma were recorded for all participants at the end of the testing session. The entire battery took approximately twenty-five minutes to administer.

\section{Primary cognitive outcome measures}

The CDR system has a strong factor structure that has been demonstrated in both healthy and diseased populations (Wesnes et al., 2000, 2002). The individual test scores from the battery load strongly onto four global outcome factors (Quality of memory; Speed of attention; Accuracy of attention; Speed of memory) and two sub-factors (Working memory; Secondary memory). These factors being derived through principle components analysis combined with varimax rotation. The contribution of individual task measures to each of these factors and sub factors is illustrated schematically in Fig. 1. Wesnes et al., (2000) give the following description: Quality of memory reflects participants' ability to hold and recall information from working and long term episodic memory. Speed of attention reflects the ability to focus on a particular task for a short period to the exclusion of other tasks. Accuracy of attention reflects the ability to sustain attention over the longer term. Speed of memory reflects the speed with which participants are able to identify whether or not something is held in memory. The Working memory sub factor reflects the capability of holding information temporarily in the articulatory loop and the visual-spatial sketchpad. The Secondary memory sub factor reflects the ability to hold and retrieve information from secondary memory. These factors have been previously utilised as primary outcome measures employing a range of participant populations and for a variety of pharmacological and herbal interventions e.g. (Kennedy et al., 2000, 2001; Wesnes et al., 1999; Moss et al., 2003, 2006).

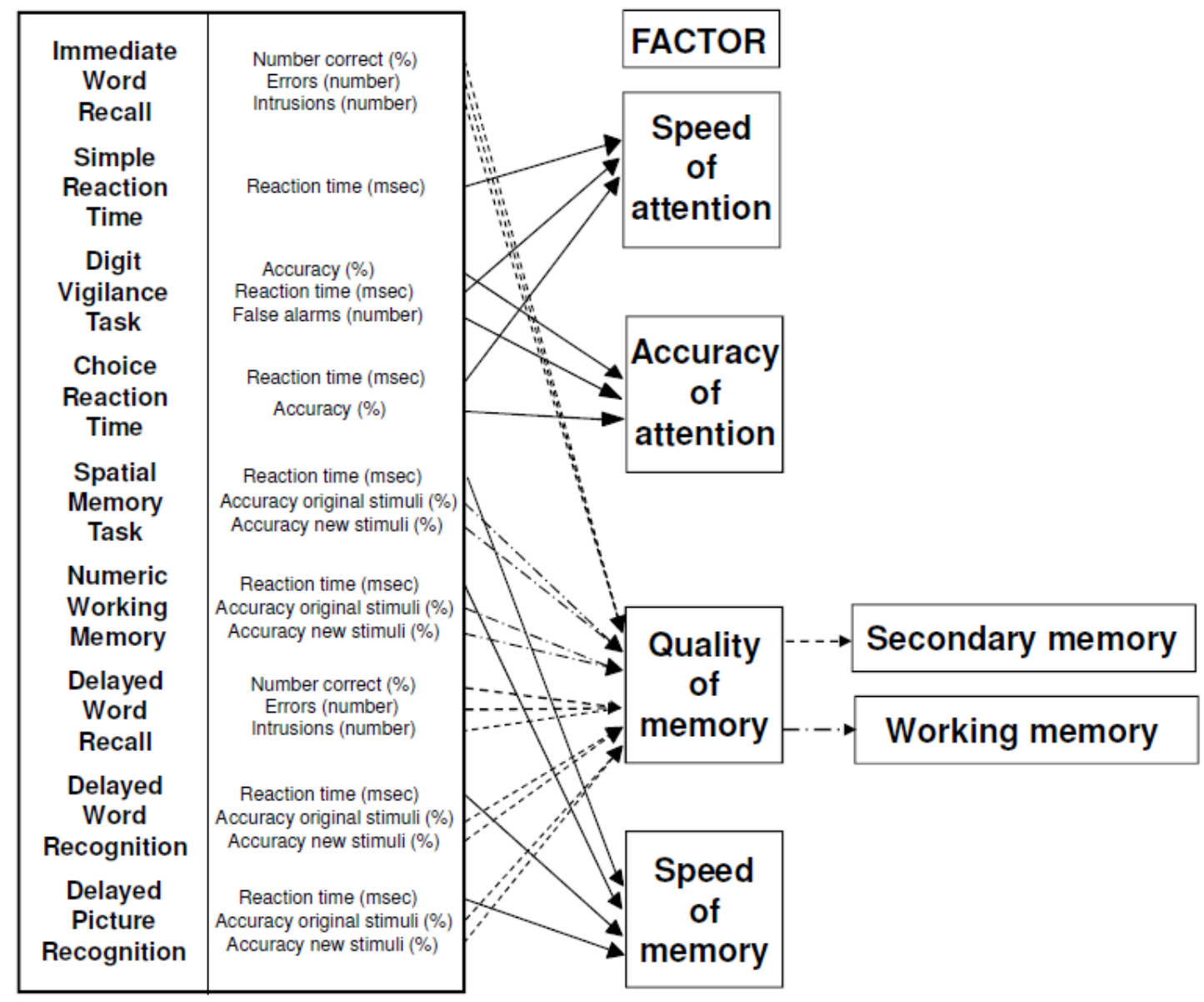

Fig. 1. Schematic representation of the CDR battery showing (from left to right) running order of tasks, individual task outcome measures and the composition of the four factors derived by factor analysis. Arrows indicate that a task outcome measure contributes to the given factor "Speed of Attention", "Accuracy of Attention", "Quality of Memory" or "Speed of Memory". Dotted lines indicate contribution to both "Quality of Memory" and to either "Working Memory" ( ) or "Secondary Memory" (---), respectively. 


\section{Subjective Mood Measure}

The Bond-Lader visual analogue scales (Bond and Lader, 1974) were employed to assess mood. The 16 visual analogue scales were combined as recommended by the authors to form three mood factors: "alert", "calm" and "content".

\section{Sensory and Expectancy Variables}

Likert scales were used to assess participants' ratings of pleasantness and strength of the drinks they consumed. The scales ranged from 1 to 5 with higher values indicating stronger ratings of the variables. At the end of the testing session participants were also asked 'what effect, if any, did you expect your drink to have on you?' and selected from 'relaxing', 'arousing' or 'no effect'.

\section{Procedure}

The study was approved by the Department of Psychology ethics committee at Northumbria University. Testing took place in a quiet cubicle in the Department of Psychology research hub. Participants were required to fast for two hours prior to taking part and refrain from consuming caffeine on the day up to the time of testing. Participants provided informed consent and were asked to complete a pre-testing mood scale. This was followed by the consumption of the drink determined by their condition. Participants were not told which condition they were in, but many recognised the taste of the teas, particularly in the Peppermint condition. Following consumption of the drink a twenty minute rest period allowed for absorption of active compounds. Participants then completed the CDR computerised test battery and the second mood scale, and the sensory and expectancy scales. Finally participants were debriefed and any questions answered. Total participation time was approximately one hour.

\section{Results}

\section{Cognition}

Data from the CDR system were analysed using SPSS version 16. Independent groups Anovas were performed followed by Tukey pairwise comparisons where significant differences were identified by the omnibus test. See also Fig. 2.

\section{Quality of Memory}

The Anova identified a significant difference between the three conditions $F(2,177)=15.19, p<$ $0.05, \eta_{p=0.14}^{2}$. Tukey post hoc comparisons revealed that the Peppermint condition produced significantly higher scores (mean $=384.5)$ than both the Chamomile condition (mean $=359.6)$ and the control condition (mean $=370.3$ ), $\mathrm{p}<0.05$ in each case. The difference between the Chamomile and control conditions approached but did not quite reach significance, $\mathrm{p}=0.051$.

\section{Secondary Memory}

The Anova identified a significant difference between the three conditions $F(2,177)=11.862$, $p<$ $0.05, \eta_{p=0.11}^{2}$. Tukey post hoc comparisons revealed that the Peppermint condition produced significantly higher scores (mean $=203.6$ ) than both the Chamomile condition (mean $=188.9)$ and the control condition (mean $=195.6), p<0.05$ in each case. The difference between the Chamomile and control conditions approached but did not reach significance, $\mathrm{p}=0.072$.

\section{Working Memory}

The Anova identified a significant difference between the three conditions $\mathrm{F}(2,177)=6.31, \mathrm{p}<$ $0.05, \eta_{p=0.07}^{2}$. Tukey post hoc comparisons revealed that the Peppermint condition produced significantly higher scores $($ mean $=180.8)$ than the Chamomile condition $($ mean $=170.7) \mathrm{p}<0.05$, but not the control condition (mean $=174.7$ ). The Chamomile and control conditions did not differ.

\section{Speed of Memory}

The Anova identified a significant difference between the three conditions $F(2,177)=9.73, p<$ $0.05, \eta_{p=0.09}^{2}$. Tukey post hoc comparisons revealed that the Peppermint condition produced significantly faster responses $($ mean $=3626 \mathrm{msec}$ ) than both the Chamomile condition (mean $=4131$ msec) and the control condition (mean $=3982$ msec), $\mathrm{p}<0.05$ in each case. The Chamomile and control conditions did not differ.

\section{Accuracy of Attention}

The Anova identified no significant difference between the three conditions $F(2,177)=2.78, p$ > $0.05, \eta_{p=0.03}^{2}$.

\section{Speed of Attention}

The Anova identified a significant difference between the three conditions $F(2,177)=5.51, p<$ $0.05, \eta_{p=0.06}^{2}$. Tukey post hoc comparisons revealed that the Peppermint condition produced significantly faster responses $($ mean $=1190 \mathrm{msec}$ ) than the Chamomile condition (mean $=1267 \mathrm{msec}$ ) $\mathrm{p}<0.05$, but not the control condition (mean $=$ 

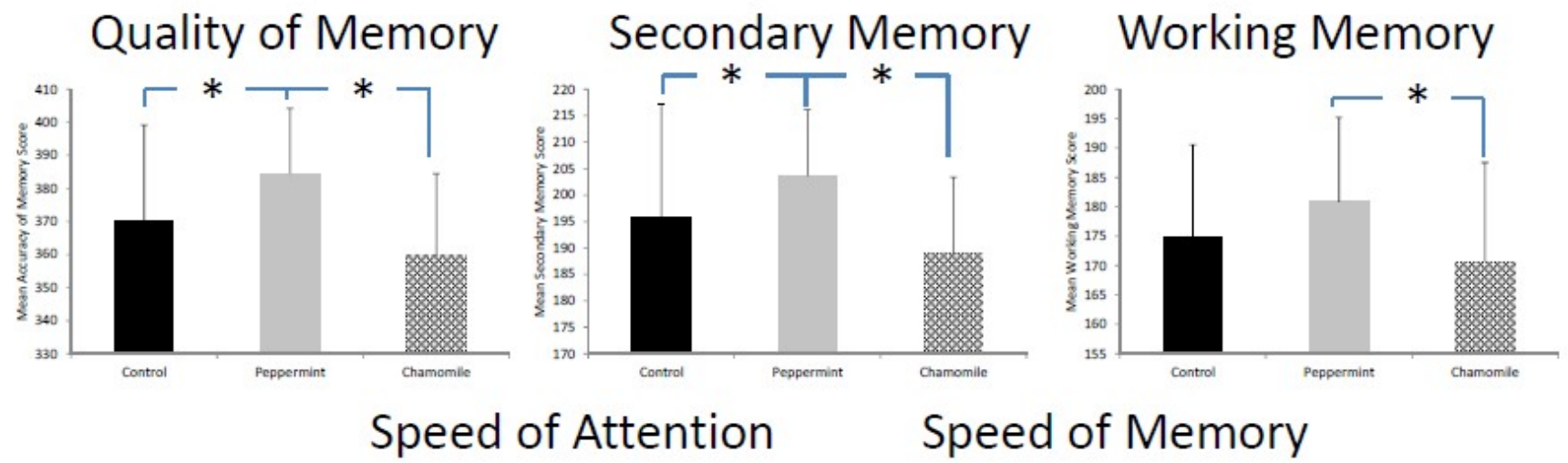

\section{Speed of Memory}
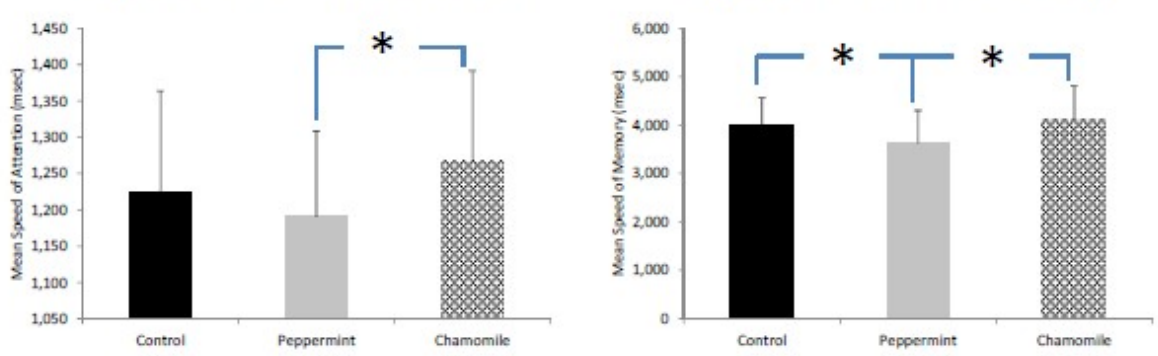

Fig. 2. Mean scores of the three conditions for the cognitive factors that reached significance. Error bars represent standard deviations. Significant pairwise comparisons are indicated as *.

$1223 \mathrm{msec})$. The Chamomile and control conditions did not differ.

\section{Mood}

Initial analyses revealed no differences between conditions at pre-test baseline for the three mood variables Alert, Content and Calm, $\mathrm{F}<1$ in each case. To analyse the mood data further the pretest ratings were subtracted from the post-test ratings to produce change scores for each of the three variables, such that positive values indicate an increase in that variable over the period of the testing session. See also Fig. 3.

\section{Alert}

The Anova revealed a significant difference between conditions for the change in alertness, $\mathrm{F}(2,177)=13.90, \mathrm{p}<0.05, \eta_{p=0.14}^{2}$. Tukey post hoc comparisons indicated that the Peppermint condition produced an increase in alertness (mean $=11.2$ ) that was significantly greater than that for the control condition (mean $=3.8$ ), which in turn was significantly different to an observed decline in alertness in the Chamomile condition (mean = $-4.1), \mathrm{p}<0.05$ in all cases.

\section{Content}

The Anova revealed no significant differences between the conditions for the change in contentedness scores, $\mathrm{F}(2,177)=0.951, \mathrm{p}=0.388$, $\eta_{p=0.01 \text {. }}^{2}$.

\section{Calm}

The Anova revealed a significant difference between conditions for the change in calmness, $\mathrm{F}(2,177)=8.57, \mathrm{p}<0.05, \eta_{p=0.08}^{2}$. Tukey post hoc comparisons indicated that the Chamomile condition produced an increase in Calmness (mean $=5.7)$ that was significantly different to decreases observed in both the control condition (mean $=-4.0$ ), and the Peppermint condition (mean $=-8.9) \mathrm{p}<0.05$ in all cases.

\section{Sensory and Expectancy Variables Pleasant}

The Anova identified a significant difference between groups for the rating of the drinks' pleasantness $\mathrm{F}(2,177)=56.32, \mathrm{p}<0.05, \quad \eta_{p=0.39}^{2}$. Tukey post hoc comparisons revealed that both the Peppermint (mean $=3.43$ ) and the Chamomile (mean $=3.72)$ teas were rated as significantly more pleasant than the hot water $($ mean $=1.95)$.

\section{Strong}

The Anova identified a significant difference between groups for the rating of the drinks' strength $\mathrm{F}(2,177)=103.58, \mathrm{p}<0.05, \eta_{p=0.54}^{2}$. Tukey post hoc comparisons revealed that the Peppermint tea (mean $=3.62$ ) was rated as stronger than the Chamomile (mean $=2.97$ ) which in turn was rated as stronger than the hot water $($ mean $=1.43)$. 

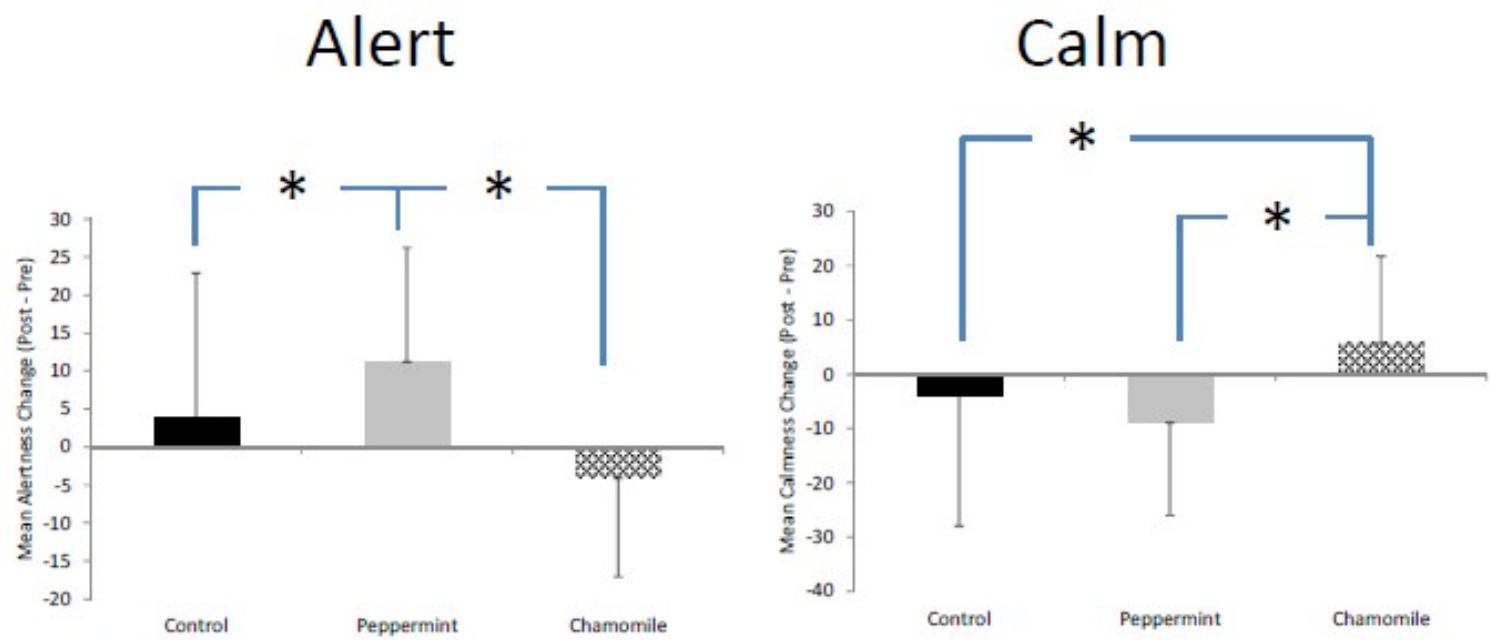

Fig. 3. Mean change in mood scores for the dimensions that reached significance. Error bars represent standard deviations. Significant pairwise comparisons are indicated as *.

\section{Expectancy}

A Chi square test of independence indicated that the expected effect of the drink was dependent on which drink was consumed, $X^{2}(4)=32.42, p<0.05$, with nearly all participants in the water condition selecting 'no effect'. Given the large impact of this on the analysis a comparison was made of the relative frequencies for just the two tea conditions, and this also proved to be significant, $X^{2}(4)=6.99$, $p<0.05$. Comparison of the frequencies indicated that more participants in the Peppermint condition $(\mathrm{N}=17)$ expected an arousing effect than did in the Chamomile condition $(\mathrm{N}=6)$, and conversely more participants in the Chamomile condition $(\mathrm{N}=16)$ expected a relaxing effect than did in the Peppermint condition $(\mathrm{N}=10)$. It is worth noting that around sixty percent of the participants in these two conditions also indicated no expected effect of drinking the tea.

\section{Correlations}

Pearson correlations revealed no significant relationships between the mood measures and cognitive performance factors, $p>0.05$ in all cases.

\section{Discussion}

The impact of the acute consumption of Chamomile and Peppermint teas on cognition and mood was largely in line with predictions made and based on the extant literature and historically recorded reputations of the two herbs. Peppermint improved both long term and working memory accuracy and speed compared to controls, whilst Chamomile had an impairing effect on memory that approached but did not quite reach statistical significance. For the factors that assessed attention no effect was observed for accuracy, and a significant difference found for speed between the two tea conditions but not compared to controls. With regard to the assessments of mood, Peppermint produced an increase in alertness whereas Chamomile led to a decrease; a pattern that was reversed for the calmness measure.

The observed effect sizes reported here are consistently small to medium for the cognitive performance factors, with condition explaining between three and fourteen percent of the variance, with the highest value being for the combined overall quality of memory factor. These are largely comparable to the effects reported previously for the beneficial impact of chronic peppermint consumption on a range of physiologic and performance measures during exercise (Meamarbashi and Rajabi, 2013a). The authors attribute these effects to a number of possible mechanisms, two of which are particularly relevant here. Firstly is the increase in glucose metabolism observed following peppermint supplementation. Any such effect - should it occur in the brain - would potentially improve cognitive performance in a manner consistent with previous demonstrations of the impact of the acute administration of glucose (Owens and Benton, 1994) and oxygen (Moss and Scholey, 1996) the human body's fuels. Indeed, the observed enhancements of memory accuracy and speed reported here reflect the aspects of cognition positively affected by acute administration of these 'fuels'. Meamarbashi and Rajabi's second suggestion is based around the stimulating effect of Peppermint on the central nervous system which is also manifest subjectively and this might reduce perceived workload and so improve performance. Were this to be the case a correlation between alertness and cognitive task scores would be expected to be observed. The clear lack of any such correlation here suggesting that a simple stimulation effect is not responsible for the observed cognitive enhancement.

Previous research has also investigated the effects of acute exposure to the aroma of Peppermint and Chamomile on cognition and 
mood. Specifically, the same computerised test battery has been employed in two studies involving one each of these aromas. Moss et al., (2008) administered the tests to healthy adults in a cubicle infused with the aroma of Peppermint essential oil and found that overall quality of memory was significantly improved compared to no aroma controls, and long term memory, working memory and speed of attention were all significantly better in the Peppermint condition when compared to the 'sedative' aroma of Ylang Ylang. Effect sizes were similar to those reported here. However, alertness was affected less than in the current study and the pre to post change did not differ between the Peppermint and control conditions to the extent that it did here. The lack of any correlations between mood and performance might be taken as supporting evidence that changes in subjective state are not at the root of enhancements in cognition - an argument made by Moss and colleagues.

The effects of Chamomile tea on the 'calm' mood dimension were predicted and match the herbs historical reputation as a mild sedative and limited evidence from scientific investigation (Zick et al., 2011). The effect size was again moderate and reflects both an increase in calmness in the Chamomile condition combined with a decrease in the control and Peppermint conditions. This pattern is consistent with that observed by Moss et al., (2006) when investigating the effect of Chamomile aroma. Interestingly the impact on cognition is less consistent. Employing the same battery of tests, no significant differences were reported between the control and Chamomile conditions for the memory factors except in the 'expected sedation' condition where participants were provided with information suggesting that their performance would be likely to be impaired and not to worry about this as any effect would dissipate shortly after completion of the study. In contrast, and although expectancies were not manipulated in the current study, the quality of memory and secondary memory factor scores bordered on significant impairment compared to controls. Equally, Moss and colleagues reported a significant impairment for accuracy of attention in the Chamomile condition. Something that was not apparent in the current study's data. The exact reason for this inconsistency is not clear but may represent differences in the bioavailability of active compounds when administered orally compared to ambient aroma. Inhalation of active compounds is generally the 'cleanest' route of administration as anything absorbed is carried straight to the brain without any first pass metabolism in the gut or liver. However, it might be the case that some active compounds in Chamomile are not volatile to be absorbed by this route but are present in the tea infusion and may be absorbed via the gut leading to greater impact of the herb overall.
The subject of bioavailability brings us neatly to consider the mechanism(s) that may underpin the activity observed here and elsewhere for herbal extracts and aromas of essential oils. With all extracts of aromatic plants there exist significant and distinguishable characteristic aromas. Indeed it is this aspect that led to human interest in these plants in the first instance, and today metabolic engineering is undertaken to try and enhance the aromas of mass produced flora and food crops (Dudareva and Pichersky, 2008). Given the potential importance of the nature of the aroma is it possible that some sensory property or qualia of the aroma itself might be responsible for the observed psychological effects? Certainly aromas have great strength in their ability to stimulate the recall of memories long since past, something often termed the 'Proust effect' after the writer who so eloquently described his experience. However, there is no evidence to be gleaned from the current study that the plant based aromas are responsible. Although the teas do give off characteristic aromas it seems unlikely that the observed pattern of effects was a consequence of these rather than the consumption of the infusions. The extent of the aroma inhaled when sipping from a cup is likely too small to impart a significant effect. Research has shown that boiled water infusions of dried Peppermint leaf contain approximately $21 \%$ of the main volatile compounds found in the essential oil, but that the proportions of individual components are not consistently the same (Duband et al., 1991). Active compounds identified in Peppermint include menthol, menthone, 1,8 - cineole and rosmarinic acid, the latter two of which have been shown to possess cholinergic agonist properties via the inhibition of acetylcholine esterase activity (Perry et al., 2003; Orhan et al., 2008). Such a mechanism could underpin the cognitive effects observed here and elsewhere as acetylcholine is the fundamental memory neurotransmitter, whilst the dopaminergic influence of menthol and menthone might be independently reflected in the subjective ratings of alertness. Animal models demonstrate that these compounds increase movement (Umezu, 2009) an aspect of behaviour which has previously been measured in combination with or as a proxy for alertness in human studies (Ivancic et al., 1997). The observation that subjective mood and objective performance do not correlate might at first sight be surprising but the possibility of independent pharmacological mechanisms underpinning the two aspects provides an intriguing explanation.

The major active compounds in Chamomile are $\beta$-farnesene, farnesol, chamazulene, and $\alpha$ bisabolol and these are responsible for the antiinflammatory, antiseptic, antiplogistic, and spasmolytic properties ascribed to the plant (Singh et al., 2011). However, more than 120 chemical constituents have been identified in chamomile flowers as secondary metabolites, including 28 
terpenoids, 36 flavonoids, and 52 additional compounds with potential pharmacological activity, and although studies have investigated the potential for anxiolytic (Amsterdam et al., 2009), anti-stress and anti-depressant (Pinto et al., 2008) and sedative properties (Kesmati et al, 2008) the exact pharmacological mechanism has not been specified although Kesmati and colleagues provide evidence for the presence of benzodiazepine ligands in Chamomile extracts. A potential candidate for the impact on cognition is the flavonoid compound apigenin that has been shown to inhibit the major excitatory neurotransmitter glutamate in rat hippocampus (Chang et al., 2015). Such an effect may be related to the observed slowing of memory and attention in the current study following Chamomile tea consumption. Furthermore, the data presented here that indicate no correlation between calmness and cognitive performance measures suggest the mild cognitive impairment effects are independent of the more robust sedative properties observed. This would appear reasonable if the effects are differentially mediated.

In conclusion the results from the current study reinforce the reputation of aromatic herbs to have differential beneficial impacts on human behaviour. Small benefits to cognition and increased alertness following consumption of peppermint tea, and the calming effect of Chamomile support data previously published. The diversity of plants constituents based on geography, climate and processing continue to provide challenges to research in this area, but the data continue to encourage further efforts to be made.

\section{Competing Interest}

The authors declare that they have no competing interests.

\section{Authors' contributions}

MM designed and coordinated the study. RJ, LM and RC conducted the data collection and initial analysis. KW designed and constructed the cognitive assessment system and interpreted the data analysis. All authors contributed to the writing of the manuscript. MM produced the final version and corrections of the manuscript. All authors read and approved the final content of the manuscript. MM submitted the final script through his account.

\section{Acknowledgements}

The authors are grateful to the faculty of Health and Life Sciences at Northumbria University, Newcastle, UK for supporting this work.

\section{References}

Amsterdam, J. D., Li, Y., Soeller, I., Rockwell, K., Mao, J. J., and Shults, J. 2009. A randomized, double-blind, placebo-controlled trial of oral Matricaria recutita (chamomile) extract therapy for generalized anxiety disorder. J Clin Psychopharmacol, 29. doi: 10.1097/JCP.0b013e3181ac935c

Bent, S., and Ko, R. 2004. Commonly used herbal medicines in the United States: a review. The American Journal of Medicine, 116(7), 478-485. doi: doi: 10.1016/j.amjmed.2003.10.036

Bond, A., and Lader, M. 1974. The use of analogue scales in rating subjective feelings. British Journal of Medical Psychology, 47(3), 211-218.

Burleigh, S., and Armstrong, C. 1997. On the scent of a useful therapy: Arotheraphy has many champions in dementia care but there has been little research to evaluate its effects. Journal of Dementia Care, 5, 2123.

Chang, C. Y., Lin, T. Y., Lu, C. W., Wang, C. C., Wang, Y. C., Chou, S. S. P., and Wang, S. J. 2015. Apigenin, a natural flavonoid, inhibits glutamate release in the rat hippocampus. European Journal of Pharmacology, 762, 72-81. doi: 10.1016/j.ejphar.2015.05.035

Duband, F., Carnat, A., Carnat, A., Petitjean-Freytet, C., Clair, G., and Lamaison, J. 1991. [Aromatic and polyphenolic composition of infused peppermint, Mentha x piperita L.]. Paper presented at the Annales pharmaceutiques francaises, 50(3): 146-55.

Dudareva, N., and Pichersky, E. 2008. Metabolic engineering of plant volatiles. Current Opinion in Biotechnology, 19(2), 181-189. doi: 10.1016/j.copbio.2008.02.011

Ernst, E. 2000. Prevalence of use of complementary/alternative medicine: a systematic review. Bulletin of the World Health Organization, 78, 258-266.

Ford, A. C., Talley, N. J., Spiegel, B. M. R., Foxx-Orenstein, A. E., Schiller, L., Quigley, E. M. M., and Moayyedi, P. 2008. Effect of fibre, antispasmodics, and peppermint oil in the treatment of irritable bowel syndrome: systematic review and meta-analysis. BMJ, 337. doi: $10.1136 /$ bmj.a2313

Heimes, K., Hauk, F., and Verspohl, E. J. (2011). Mode of action of peppermint oil and (-)-menthol with respect to 5-HT3 receptor subtypes: binding studies, cation uptake by receptor channels and contraction of isolated rat ileum. Phytotherapy Research, 25(5), 702708. doi: $10.1002 / p t r .3316$

Ivancic, M. T., Barrett, G. T., Simonow, A., and Kimberly, A. 1997. A replication to increase happiness indices among some people with profound multiple disabilities. Research in Developmental Disabilities, 18(1), 79-89. doi: 10.1016/S0891-4222(96)00039-X

Jasira, M., Sai-Sailesh, K.\& Mukkadan, J.K. 2013. Oral administration of peppermint in Wistar albino rats: Memory boosting and regaining. Indonesian Jornal of Biomedical Sciences, 7(1): 23-26.

Kakuta, H., Yano-Kakuta, E., and Moriya, K. 2007. Psychological and Physiological Effects in Humans of Eating Chamomile Jelly. Acta Hortic. 749, 187-192. doi: 10.17660/ActaHortic.2007.749.20

Kennedy, D., Scholey, A., and Wesnes, K. 2001. Dose dependent changes in cognitive performance and mood following acute administration of Ginseng to healthy young volunteers. Nutr Neurosci, 4(4), 295310.

Kennedy, D. O., Scholey, A. B., and Wesnes, K. A. 2000. The dose-dependent cognitive effects of acute administration of Ginkgo biloba to healthy young volunteers. Psychopharmacology, 151(4), 416-423.

Kesmati, M., Abbasi Zadeh, Z., and Mofhaddam, H. 2008. Study of benzodiazepine like effects of Matricaria recutita on morphine withdrawal syndrome in adult male rats. PakJ Med Sci, 24(5), 735-739. 
Kim, M.-J., Nam, E.-S., and Paik, S.-I. 2005. [The effects of aromatherapy on pain, depression, and life satisfaction of arthritis patients]. Taehan Kanho Hakhoe chi, 35(1), 186-194.

Kulisic-Bilusic T, Katalinic V, Dragovic-Uzelac V, Ljubenkov I, Krisko A, Dejanovic B, Jukic M, Politeo O, Pitaf G, Milos M. 2008. Antioxidant and acetylcholinesterase inhibiting activity of several aqueous tea infusions in vitro. Food Technol. Biotechnol., 46: 68-375.

Meamarbashi, A., and Rajabi, A. 2013a. The effects of peppermint on exercise performance. Journal of the International Society of Sports Nutrition, 10(1), 1-6. doi: 10.1186/1550-2783-10-15

Meamarbashi, A., and Rajabi, A. 2013b. The effects of peppermint on exercise performance. Journal of the International Society of Sports Nutrition, 10, 15-15. doi: 10.1186/1550-2783-10-15

Mills, S., and Bone, K. 2000. Principles and practice of phytotherapy. Modern herbal medicine. Edinburgh: Churchill Livingstone.

Moss, M., Cook, J., Wesnes, K., and Duckett, P. 2003. Aromas of rosemary and lavender essential oils differentially affect cognition and mood in healthy adults. International Journal of Neuroscience, 113(1), 15-38. doi: 10.1080/00207450390161903

Moss, M., Hewitt, S., Moss, L., and Wesnes, K. 2008. Modulation of cognitive performance and mood by aromas of peppermint and ylang-ylang. International Journal of Neuroscience, 118(1), 59-77.

Moss, M., Howarth, R., Wilkinson, L., and Wesnes, K. 2006. Expectancy and the aroma of Roman chamomile influence mood and cognition in healthy volunteers. International Journal of Aromatherapy, 16(2), 63-73.

Moss, M. C., and Scholey, A. B. 1996. Oxygen administration enhances memory formation in healthy young adults. Psychopharmacology, 124(3), 255-260. doi: 10.1007/bf02246665

Orhan, I., Aslan, S., Kartal, M., Şener, B., and Hüsnü Can Başer, K. 2008. Inhibitory effect of Turkish Rosmarinus officinalis L. on acetylcholinesterase and butyrylcholinesterase enzymes. Food Chemistry, 108(2), 663-668. doi: 10.1016/j.foodchem.2007.11.023

Owens, D. S., and Benton, D. 1994. The Impact of Raising Blood Glucose on Reaction Times. Neuropsychobiology, 30(2-3), 106-113.

Perry, N. S. L., Bollen, C., Perry, E. K., and Ballard, C. 2003. Salvia for dementia therapy: review of pharmacological activity and pilot tolerability clinical trial. Pharmacology Biochemistry and Behavior, 75(3), 651-659. doi: 10.1016/S00913057(03)00108-4

Pinto, S. A., Bohland, E., Coelho Cde, P., Morgulis, M. S., and Bonamin, L. V. 2008. An animal model for the study of Chamomilla in stress and depression: pilot study. Homeopathy, 97(3), 141-144. doi: 10.1016/j.homp.2008.04.001

Raudenbush, B., Grayhem, R., Sears, T. \& Wilson, I. 2009. Effects of peppermint and cinnamon odor administration on simulated driving alertness, mood and workload. North American Journal of Psychology, 11(2): 245-256.

Sánchez-Ortuño, M. M., Bélanger, L., Ivers, H., LeBlanc, M., and Morin, C. M. 2009. The use of natural products for sleep: A common practice? Sleep Medicine, 10(9), 982-987. doi: 10.1016/j.sleep.2008.10.009

Scholey, A. B., Tildesley, N. T., Ballard, C. G., Wesnes, K. A., Tasker, A., Perry, E. K., and Kennedy, D. O. 2008. An extract of Salvia (sage) with anticholinesterase properties improves memory and attention in healthy older volunteers. Psychopharmacology, 198(1), 127-139.

Shkurupiǔ, V. A., Odintsova, O. A., Kazarinova, N. V., and Tkrachenko, K. G. 2006. [Use of essential oil of peppermint (Mentha piperita) in the complex treatment of patients with infiltrative pulmonary tuberculosis]. Problemy tuberkuleza $i$ boleznei legkikh(9), 43-45.

Singh, O., Khanam, Z., Misra, N., and Srivastava, M. K. 2011. Chamomile (Matricaria chamomilla L.): An overview. Pharmacognosy Reviews, 5(9), 82-95. doi: 10.4103/0973-7847.79103

Srivastava, J. K., Shankar, E., and Gupta, S. 2010. Chamomile: A herbal medicine of the past with bright future. Molecular medicine reports, 3(6), 895901. doi: $10.3892 / \mathrm{mmr} .2010 .377$

Tate, S. 1997. Peppermint oil: a treatment for postoperative nausea. Journal of Advanced Nursing, 26(3), 543-549. doi: 10.1046/j.1365-2648.1997.t01-15-00999.x

Umezu, T. 2009. Evidence for dopamine involvement in ambulation promoted by menthone in mice. Pharmacology Biochemistry and Behavior, 91(3), 315320. doi: 10.1016/j.pbb.2008.07.017

Umezu, T., and Morita, M. 2003. Evidence for the Involvement of Dopamine in Ambulation Promoted by Menthol in Mice. Journal of Pharmacological Sciences, 91(2), 125-135. doi: 10.1254/jphs.91.125

Vickers, A., and Zollman, C. 1999. Herbal medicine. BMJ, 319(7216), 1050-1053. doi: 10.1136/bmj.319.7216.1050

Viola, H., Wasowski, C., Levi de Stein, M., Wolfman, C., Silveira, R., Dajas, F., Medina, J. H. , Paladini, A. C. 1995. Apigenin, a component of Matricaria recutita flowers, is a central benzodiazepine receptors-ligand with anxiolytic effects. Planta Med, 61. doi: 10.1055/s2006-958058

Wang, Y., Tang, H., Nicholson, J. K., Hylands, P. J., Sampson, J., and Holmes, E. 2005. A metabonomic strategy for the detection of the metabolic effects of chamomile (Matricaria recutita L.) ingestion. J Agric Food Chem, 53(2), 191-196. doi: 10.1021/jf0403282

Wesnes, K., Ward, T., Ayre, G., and Pincock, C. 1999. Validity and utility of the cognitive drug research (CDR) computerised cognitive testing system: A review following fifteen years of usage. European Neuropsychopharmacology, 9, 368.

Wesnes, K., Ward, T., McGinty, A., and Petrini, O. 2000. The memory enhancing effects of a Ginkgo biloba/Panax ginseng combination in healthy middle-aged volunteers. Psychopharmacology, 152(4), 353-361.

Wesnes, K. A., McKeith, I., Ferrara, R., Emre, M., Del Ser, T., Spano, P., Cicin-Sain, A., Anand, R., Spiegel R. Spiegel, R. 2002. Effects of rivastigmine on cognitive function in dementia with lewy bodies: a randomised placebo-controlled international study using the cognitive drug research computerised assessment system. Dementia and geriatric cognitive disorders, 13(3), 183-192.

Zeggwagh, N. A., Moufid, A., Michel, J. B., and Eddouks, M. 2009. Hypotensive effect of Chamaemelum nobile aqueous extract in spontaneously hypertensive rats. Clin Exp Hypertens, 31(5), 440-450.

Zick, S. M., Wright, B. D., Sen, A., and Arnedt, J. T. 2011. Preliminary examination of the efficacy and safety of a standardized chamomile extract for chronic primary insomnia: A randomized placebo-controlled pilot study. BMC Complementary and Alternative Medicine, 11(1), 1-8. doi: 10.1186/1472-6882-11-78 